AperTO - Archivio Istituzionale Open Access dell'Università di Torino

Mitochondrial metabolic alterations in cancer cells and related therapeutic targets

This is a pre print version of the following article:

Original Citation:

Availability:

This version is available http://hdl.handle.net/2318/1720964

since 2020-07-29T22:13:29Z

Published version:

DOI:10.1016/j.semcdb.2019.10.005

Terms of use:

Open Access

Anyone can freely access the full text of works made available as "Open Access". Works made available under a Creative Commons license can be used according to the terms and conditions of said license. Use of all other works requires consent of the right holder (author or publisher) if not exempted from copyright protection by the applicable law. 


\section{Mitochondrial metabolic alterations in cancer cells and related therapeutic targets}

Mitochondria have been regarded as the power generators of cells. Despite cancer cells have higher demand of energy than non-transformed cells, since Otto H. Warburg discovered that cancer cells have high glycolytic flux also in the presence of oxygen, the study of mitochondria metabolism and functions in cancer has been neglected for long time. Indeed, it was erroneously supposed that cancer cells have defective mitochondria, unable to meet the energy demands of transformed cells. Far from being defective, in the last twenty years it has become clear that mitochondria are often the drivers of the malignant phenotype.

Indeed, many tumors exploit also mitochondria-related pathways, such as tricarboxylic acid (TCA) cycle and the oxidative phosphorylation (OXPHOS), fueled by the fatty acid $\beta$ oxidation (FAO) and the glutaminolysis, to produce ATP and building blocks. Mitochondrial reactive oxygen species (ROS), i.e. physiological side-products of OXPHOS, are finely tuned in cancer cells, where they can act as signaling molecules, stimulating pro-proliferative, antiapoptotic and pro-invasive pathways. A controlled balance of mitochondria dynamics is also critical to support an accelerated cell proliferation and migration. Since higher is the cell metabolic plasticity, higher is the ability to adapt to unfavorable conditions - such as nutrient shortage, hypoxia, oxidative stress induced by radiotherapy or chemotherapy, cells with a high plasticity in mitochondria functions are the most favored to survive to stressors, generating aggressive and potentially therapies-resistant clones. This Special Issue is focused on the latest discoveries on the mitochondrial metabolism related to cancer develop, progression and resistance.

The physiological or pathological activation of specific mitochondrial enzymes have been related to oncogenic properties. For instance, succinate dehydrogenase (SDH) has emerged as 
a key enzyme in tumors, since it consumes succinate that promotes cell proliferation and migration. The loss of function of SDH, due to inactivating mutations, down-regulation of mRNA levels by specific miRNAs, dephosphorylation and/or deacetylation, inhibition of enzymatic activity by itaconate or by the mitochondrial chaperon Necrosis Factor ReceptorAssociated Protein (TRAP1), increases the level of succinate that inhibits several $\alpha$ ketoglutarate-dependent histone demethylase ( $\alpha$-kGDDs), producing a wide epigenetic reprogramming, activates several glycolytic enzymes via succinylation, turns off the mitochondrial metabolism, induces epithelial-mesenchimal transition (EMT) and angiogenesis. The possibility of detecting SDH in tissues and succinate in biological fluids, and of modulating SDH activity with several FDA-approved agents, paves the way to the identification of new biomarkers of tumor progression and to the use of mitochondrial-targeting treatments as new anti-cancer approaches [Dalla Pozza/Donadelli]. Very similarly to SDH, the loss of fumarate hydratase $(\mathrm{FH})$ is a pro-oncogenic factor: the consequent increase in fumarate reduces the efficiency of mitochondrial OXPHOS, promotes cell proliferation, EMT and angiogenesis via the succinylation-dependent activation of pro-oncogenic factors and/or the inhibition of $\alpha$-kGDDs. Of note, both the loss of SDH and FH are considered early events in the transformation cascade, pointing out that an alteration of the physiological mitochondrial metabolism may act as an oncogenic trigger [Schmidt/Frezza]. By contrast, a third metabolite of TCA, $\alpha-k G$, acts as a potential onco-suppressor: contrarily to succinate and fumarate, it activates $\alpha$-kGDDs promoting opposite epigenetic reprogramming. Moreover, $\alpha-k G$ activates the prolyl hydroxylases responsible for the stabilization of collagen matrix and the degradation of Hypoxia Inducible factor-1 $\alpha$ (HIF-1 $\alpha)$ and Nuclear Factor-kB (NF-kB), and acts as a ROS scavenger, contrasting several pro-proliferative and pro-invasive pathways [Abla/Porcelli]. Consistently, $\alpha-k G$ supplementation has been regarded as an anti-cancer strategy. On the other hand, by fueling the TCA, $\alpha-k G$ supplementation has the potential threaten to increase 
succinate and fumarate. Only the exact knowledge of the relative activities of SDH, FH and isocitrate dehydrogenase in a specific tumor can orient towards the use of TCA inhibitors or stimulators in order to achieve anti-tumor effects.

Also glutamine is at the same time an anaplerotic metabolite of TCA, a source of carbon building block in proliferating cells and a signaling molecule, that increases the activity of mTOR, c-myc and c-jun oncogenic pathways, by transcriptional and post-translational mechanisms. Consistently, the down-regulation of glutaminase is often associated to reduced proliferation and migration of cancer cells. For this reason, glutaminase inhbitors have been widely explored as anti-proliferative strategy, and look particularly promising against glutamine-addicted tumors [Mates/Mazurek].

Not only changes in mitochondrial enzymes, but also in co-factors synthesized de novo in mitochondria have an impact on tumor progression. For instance, lipoic acid, the cofactor of pyruvate dehydrogenase and $\alpha-\mathrm{kG}$ dehydrogenase complexes, and coenzyme Q10, the electron shuttle between complex I and III, are strong anti-oxidant metabolites. Thanks to the ROS scavenging, they prevent the pro-proliferative and invasive phenotype induced by mitochondrial ROS. Both products, in combination with chemotherapy, have increased the overall survival of patients with metastatic cancers, suggesting that stimulating mitochondrial metabolism and buffering ROS are adequate strategies to prevent cell proliferation and migration in vivo [Thapa/Dallmann].

On the other hand, a high inter-patients and intra-tumor variability often characterize mitochondrial metabolism, making difficult designing a comprehensive picture of the reprogramming in mitochondria metabolism occurring in tumors. For instance, in non small cell lung cancer, the acetyl-coenzyme A entering the TCA cycle is generated from glucose in well-oxygenated areas and from fatty acids, lactate or amino-acids in the less-oxygenated areas of the same tumor. Curiously, glutamine, which is used by several tumors as fuel of 
mitochondrial metabolism, has a minor role in non small cell lung cancer in vivo, although it is effectively consumed in vitro [Maiem/Munoz-Pinedo]. This example highlights the need of a personalized mapping of mitochondrial metabolism in each patient, if we would like to pursue the goal of identifying the prevalent metabolic fluxes and the most suitable mitochondrialtargeting agents in each patient. The plasticity of mitochondria metabolism also characterizes the tumor progression in the same patient, and is consequent to the progressive accumulation of mutations. For instance, while normal colon epithelial cells rely on the aerobic oxidation of glucose, $c$-myc amplification and/or KRAS mutations - two early events during epithelium transformation - shifts this scenario towards a glycolytic and glutaminolytic-dependent metabolism. If this is the predominant trait or primary colon cancers, metastatic cells further reprogram their metabolism, depending on the oxygen levels of the secondary localizations. Therefore, the use of metabolic modifiers targeting mitochondria, as adjuvant agents of chemotherapy and targeted-therapies, may produce paradoxically opposite effects, depending on tumor stage and site [La Vecchia/Sebastian].

The ever changing conditions of tumor microenvironment (TME) have an important role in training mitochondria of cancer cells to rapidly reprogram their metabolisms. The paradigmatic symbiosis between lactate- and glutamine-producing cancer associated fibroblasts (CAFs) and lactate-fueled tumor cells is one example on how mitochondria of cancer cells may obtain ATP by enhancing the enter of lactate and glutamine carbons, abundant in hypoxic TME, in the TCA/OXPHOS fluxes. Similarly, breast cancer cells trigger the lipolysis of stromal adipocytes and obtain energy by the oxidation of the released fatty acids. Of note, also mitochondrial DNA can be transferred by CAFs to cancer cells, via extracellular vesicles: this is another intriguing mechanism by which stromal cells increase the efficiency of mitochondrial metabolism, promoting cell proliferation, migration and EMT [Bacci/Chiarugi]. The bidirectional interplay between mitochondrial metabolites of immune-infiltrating cells and cancer cells also tips the 
balance between immune-evasion and immune-eradication of the tumor. On the one hand, an acute release of mitochondrial danger-associated molecular pattern (mtDAMP) molecules, such as mitochondrial ROS, DNA, ATP and cardiolipin, from cancer cells promote their immunogenic death by recruiting dendritic cells, inducing tumor cell phagocytosis, activating anti-tumor $\mathrm{CD}^{+} \mathrm{T}$-cytotoxic lymphocytes. In advanced stages, however, the prolonged release of mtDAMPs, the depletion of aminoacids essential for lymphocyte proliferation as arginine and glutamine, the release of succinate, fumarate and itaconate, that favor the M2-polarization of tumor associated macrophages (TAMs), lead to the functional exhaustion of immuneinfiltrating cells. Increasing or inhibiting the mitochondrial metabolism may thus produce completely opposite effects on tumor immune-surveillance [Kopecka/Riganti]. The reciprocal influences between cancer cells, CAFs, adipocytes, immune-infiltrating cells, endothelial cells make extremely difficult a precise understanding of which compartment is the mainly affected by mitochondria-targeting agent. For instance, metformin, that inhibits mitochondrial complex I, acts on different components of TME. The anti-cancer effect of this drug must be considered the sum of the direct inhibition of tumor OXPHOS, and several indirect events, e.g. the inhibition of neo-angiogenic properties in endothelial cells, the inhibition of NF-kB-dependent inflammatory signals from CAFs, the prevailing M1-polarization of TAMs, the enhancement of cytotoxic properties of infiltrating lymphocytes [Kurelac/Gasparre].

The existence of a continuous metabolic reprogramming during the evolution of the same tumor and/or within the same tumor, due to the complex interactions with TME, requires new in vitro systems and assays to study tumor metabolism in a real time fashion and in the closest way to mimic the tumor-TME network existing in vivo. To this aim, organoids and organotypic 3D-cultures represent important technological advancements that allow the study of mitochondrial metabolism and functions in live tumor cells, surrounded by a TME similar to that of the original tumor [Silva-Almeida/Ewart]. 
Besides alterations in metabolism, changes in mitobiogenesis, mitophagy, mitochondrial fusion and fission also impact on tumor development and progression. Very often, specific alterations in oncogene or onco-suppressor genes affect mitochondrial functions. For instance, wild-type TP53 induces mitobiogenesis and promotes OXPHOS, while mutated TP53 up-regulates the glycolytic flux in cancer cells. By contrast, if present at germline level, mutated TP53 increases the mitochondrial functions, by upregulating the Mitochondrial Transcription Factor A (TFAM), the Complex IV component Cyto-chrome c Oxidase 2 (SCO2) and the Citrate carrier, which is important for TCA fueling. On the basis of these findings, the use of mitochondrial metabolism inhibitors may be considered an adjuvant treatment option in TP53-mutated patients [Blandino/Di Agostino]. Also Rel-A, a component of NF-kB, impacts on mitochondrial metabolism in a TP53-dependent way. Indeed, in cancer cells with wild-type TP53, Rel-A is maintained outside the mitochondria where it up-regulates an OXPHOSoriented metabolism. In mutated TP53-cells, Rel-A translocates to mitochondria where it turns off the efficient OXPHOS, while the anaerobic glycolysis and the generation of lactate. Lactate is taken up by CAFs where if activates NF-kB- and HIF-1 $\alpha$-dependent pro-survival and invasive programs. NF-kB also affects mitochondria dynamics, exploiting multiple feedforward loops. On the one hand, NF-kB activity promotes mitochondria fission and reduces mitochondrial energetic efficiency; on the other hand, ROS generated by inefficient mitochondria activate NF-kB, maintaining high the rate of mitochondrial fission. Therein, NF$\mathrm{kB}$ inhibitors are promising alterative to mitochondrial metabolism inhibitors in reducing oncogenesis and invasion [Capece/Zazzeroni].

Multiple molecular circuitries also link mitochondrial metabolism and autophagy, because mitochondria are both inducers or targets of authophagy. Metabolites such $\alpha$-ketoglutarate, FAO products, mitochondrial ROS, as well as phospholipids and ion channels localized in the 
Mitochondria-Associated endoplasmic reticulum (ER) Membrane (MAM), promote the assembly of the autophagic machinery. By removing damaged mitochondria and recycling metabolic precursors, mitophagy is a driving force in promoting cell survival and resistance to exogenous stressors in several cancers. Targeting $\mathrm{K}^{+}$and $\mathrm{Ca}^{++}$channels of MAM has been proposed as a novel anti-cancer strategy, able to induce $\mathrm{Ca}^{++}$-dependent mitochondrial derangement, autophagy alteration and cell death [Ferro/Brisson]. An impaired coupling between OXPHOS and ATP synthesis, an increased production of mitochondrial ROS, an increased burden of unfolded mitochondrial proteins, all trigger mitophagy and semescence. TP53 appears once again to dictate the linkage between mitochondrial metabolism and senescence: perturbations of mitochondrial energy metabolism activate p53/p21-dependent pathways inducing senescence and limiting mitophagy by preventing the recruitment of Parkin 1, while TP53-mutated tumors less frequently enter into a senescent phenotype notwithstanding the presence of damaged mitochondria [Abate/Caraglia].

Given their high plasticity, mitochondria are critical in the process of adaptation to stressors, leading to cell transformation and/or tumor growth notwithstanding unfavorable conditions. For instance, environmental carcinogens such as tobacco-derived nitrosamines, determine a metabolic reprogramming that induce irreversible damages and cell death, or cell survival and transformation. The activation of mitochondrial metabolism and anti-oxidant enzymes are critical in tipping the balance towards nitrosamine-induced tumorigenesis [Rossignol...da rivedere con la versione rivista].

ER stress is another condition often experimented by solid tumors exposed to hypoxia, nutrient deprivation, radiotherapy or chemotherapy. Cells surviving ER stress mount an adaptive response in MAM compartment, maintaining a mitochondria-ER coupling that allows the mitochondrial inward flux of $\mathrm{Ca}^{++}$. Such moderate increase in $\mathrm{Ca}^{++}$stimulates OXPHOS and 
ATP synthesis, buffers the ROS levels, and activates an anti-apoptotic response mediated by Bcl-2 members. $\mathrm{Ca}^{++}$releasing channels are controlled by the activation of K-RAS, Akt/mTOR pathways and/or by mutations in PTEN, suggesting that alterations in oncogenic or oncosuppressor genes mediate these metabolic adaptation and pro-survival response during ER stress [Rimessi/Pinton].

Anticancer drugs are among the main stressors that cancer cells must deal with. Mitochondria have a key role in promoting the survival to chemotherapy and targeted therapies, consequentially determining a drug resistant phenotype. For instance, doxorubicin efficacy is drastically reduced in cells with an active mitochondrial metabolism, able to produce huge amounts of ATP that fuels the drug efflux transporters belonging to the ATP Binding Cassette (ABC) transporters. Of note, cells equipped with both active mitochondrial metabolism and cytosolic glycid-catabolyzing pathways (namely glycolysis and pentose phosphate pathway, PPP) are even more resistant to doxorubicin. Besides providing ATP, functioning mitochondria characterized by efficient OXPHOS flux limit the production of mitochondrial ROS. Since part of doxorubicin toxicity is due to the increase of ROS above "harmful" thresholds, the efficient oxygen consumption within the mitochondria may prevent such increases in ROS levels. In parallel, the efficient production of reductive equivalents such as NADPH and GSH via the PPP, further prevents the raise in intracellular ROS levels, contributing to drug resistance [Capeloa/Sonveaux]. A mitochondrial metabolic reprogramming is also crucial in the response to BRAF inhibitors. Active BRAF promotes anaerobic glycolysis, but melanoma cells exposed to Vemurafenib gradually increase the TCA-/OXPHOS-based metabolism and mitobiogenesis. A higher mitochondrial metabolism must be sustained by an adequate supply of enzymes and cofactors, such as NAD ${ }^{+}$. Intriguingly, the metabolic shift induced by Vemrafenib is associated with the overexpression of nicotinamide phosphoribosyltransferase (NAMPT), the limiting enzyme in $\mathrm{NAD}^{+}$biosynthesis, that is promoted by BRAF oncogenic signaling and is 
associated with increased cell proliferation, migration and Vemurafenib resistance. Since NAMPT is a druggable enzyme, NAMPT inhibitors can be considered as indirect blockers of mitochondrial metabolism. By doing so, they may limit tumor progression and drug resistance [Audrito/Deaglio].

The metabolic symbiosis between tumor and TME cells is an important factor determining drug resistance. For instance, the active fueling of TCA cycle of cancer cells with the lactate produced by CAFs has been associated to chemoresistance, because it expands a population of dormant and drug-resistant cells characterized by a high OXPHOS-based metabolism. Mitochondrial inhibitors may eradicate dormant populations and restore chemosensitivity [Linden/Corbet]. Although we are moving towards a strongly personalized treatment in the oncological research, a lot of efforts are also put in drug repurposing. With their multifaceted metabolism and functions, mitochondria are the targets of several FDA-approved drugs, including the antidiabetic drug metformin, the antimicrobial drugs atovaquone, chloramphenicol, doxycycline, itraconazole and ivermectin, that inhibit OXPHOS and ATP synthesis. In parallel, several mitochondrial effects of chemotherapeutic drugs originally designed to hit other targets, such as doxorubicin, cisplatin and tamoxifene, have recently emerged. The first clinical trials using association of mitochondria-targeting/repurposed drugs and chemotherapy have produced encouraging results [Aminzadeh-Gohari/Kofler], paving the way to new possible combination treatments for oncological patients.

Far from being neglected organelles in tumors, mitochondria should be considered versatile mediators and therapeutic targets in cancer initiation, progression and resistance. In this perspective, we believe that studies on mitochondria will receive a predominant attention in the field of cancer cell biology in the next future.

\section{References}


[1] E. Dalla Pozza, I. Dando, R. Pacchiana, E. Liboi, M.T. Scupoli, M. Donadelli, M.

Palmieri. Regulation of succinate dehydrogenase and role of succinate in cancer. Semin Cell

Dev Biol. (2019) pii: S1084-9521(19)30056-4. doi: 10.1016/j.semcdb.2019.04.013.

[2] C. Schmidt, M. Sciacovelli, C. Frezza. Fumarate hydratase in cancer: A multifaceted tumour suppressor. Semin Cell Dev Biol. (2019) pii:S1084-9521(18)30202-7. doi:

10.1016/j.semcdb.2019.05.002.

[3] H. Abla, M. Sollazzo, G. Gasparre, L. Iommarini, A.M. Porcelli. The multifaceted contribution of $\alpha$-ketoglutarate to tumor progression: An opportunity to exploit? Semin Cell Dev Biol. (2019) pii: S1084-9521(18)30171-X. doi:10.1016/j.semcdb.2019.05.031.

[4] M.J. Matés, F.J. Di Paola, J.A. Campos-Sandoval, S. Mazurek, J. Márquez. Therapeutic targeting of glutaminolysis as an essential strategy to combat cancer. Semin Cell Dev Biol. (2019) pii: S1084-9521(19)30073-4. doi:

10.1016/j.semcdb.2019.05.012.

[5] M. Thapa, G. Dallmann. Role of coenzymes in cancer metabolism. Semin Cell Dev Biol. (2019) pii: S1084-9521(19)30070-9. doi: 10.1016/j.semcdb.2019.05.027

[6] B. Majem, E. Nadal, C. Muñoz-Pinedo. Exploiting metabolic vulnerabilities of Non small cell lung carcinoma. Semin Cell Dev Biol. (2019). pii:S1084-9521(18)30186-1. doi: 10.1016/j.semcdb.2019.06.004.

[7] S. La Vecchia, C. Sebastián. Metabolic pathways regulating colorectal cancer initiation and progression. Semin Cell Dev Biol. (2019) pii:S1084-9521(18)30168-X. doi: 10.1016/j.semcdb.2019.05.018.

[8] M. Bacci, L. Ippolito, L. Magnelli, E. Giannoni, P. Chiarugi. Stromal-induced mitochondrial re-education: Impact on epithelial-to-mesenchymal transition and cancer aggressiveness. Semin Cell Dev Biol. (2019). pii:S1084-9521(18)30218-0. doi: 10.1016/j.semcdb.2019.05.009. 
[9] J. Kopecka, E. Gazzano, B. Castella, I.C. Salaroglio, E. Mungo, M. Massaia, C. Riganti. Mitochondrial metabolism: Inducer or therapeutic target in tumor immune-resistance? Semin Cell Dev Biol. 2019 May 18. pii: S1084-9521(18)30164-2. doi:

10.1016/j.semcdb.2019.05.008.

[10] I. Kurelac, N. Umesh Ganesh, M. Iorio, A.M. Porcelli, G. Gasparre G. The multifaceted effects of metformin on tumor microenvironment. Semin Cell Dev Biol. (2019) pii: S10849521(19)30077-1. doi: 10.1016/j.semcdb.2019.05.010.

[11] C. Silva-Almeida, M.A. Ewart, C. Wilde. 3D gastrointestinal models and organoids to study metabolism in human colon cancer. Semin Cell Dev Biol. (2019) pii: S10849521(19)30079-5. doi: 10.1016/j.semcdb.2019.05.019.

[12] G. Blandino, F. Valenti, A. Sacconi, S. Di Agostino. Wild type- and mutant p53 proteins in mitochondrial dysfunction: emerging insights in cancer disease. Semin Cell Dev Biol. (2019) pii: S1084-9521(18)30163-0. doi:10.1016/j.semcdb.2019.05.011.

[13] D. Capece, D. Verzella, B. Di Francesco, E. Alesse, G. Franzoso, F. Zazzeroni. NF-кB and mitochondria cross paths in cancer: mitochondrial metabolism and beyond. Semin Cell Dev Biol. (2019) pii: S1084-9521(18)30183-6. doi:10.1016/j.semcdb.2019.05.021.

[14] F. Ferro, S. Servais, P. Besson, S. Roger, J.F. Dumas, L. Brisson. Autophagy and mitophagy in cancer metabolic remodelling. Semin Cell Dev Biol. 2019 pii:S10849521(18)30169-1. doi: 10.1016/j.semcdb.2019.05.029.

[15] M. Abate, A. Festa, M. Falco, A. Lombardi, A. Luce, A. Grimaldi, S. Zappavigna, P. Sperlongano, C. Irace, M. Caraglia, G. Misso. Mitochondria as playmakers of apoptosis, autophagy and senescence. Semin Cell Dev Biol. (2019) pii:S1084-9521(18)30187-3. doi: 10.1016/j.semcdb.2019.05.022.

[16] S. Sarlak, C. Lalou, N.D. Amoedo, R. Rossignol, Metabolic reprogramming by tobacco smoke-derived nitrosamines (TSNAs) in cancer, Semin. Cell Dev. Biol. (2019). 
[17] A. Rimessi, G. Pedriali, B. Vezzani, A. Tarocco, S. Marchi, M.R. Wieckowski, C.

Giorgi, P. Pinton P. Interorganellar calcium signaling in the regulation of cell metabolism: A cancer perspective. Semin Cell Dev Biol. (2019) pii:S1084-9521(18)30182-4. doi:

10.1016/j.semcdb.2019.05.015.

[18] T. Capelôa, Z. Benyahia, L.X. Zampieri, M.C.N.M. Blackman, P. Sonveaux. Metabolic and non-metabolic pathways that control cancer resistance to anthracyclines. Semin Cell Dev Biol. (2019) pii: S1084-9521(18)30220-9. doi:10.1016/j.semcdb.2019.05.006.

[19] V. Audrito, A. Managò, F. Gaudino, S. Deaglio. Targeting metabolic reprogramming in metastatic melanoma: The key role of nicotinamide phosphoribosyltransferase (NAMPT). Semin Cell Dev Biol. (2019) pii: S1084-9521(18)30203-9. doi:

10.1016/j.semcdb.2019.05.001.

[20] C. Vander Linden, C. Corbet. Reconciling environment-mediated metabolic heterogeneity with the oncogene-driven cancer paradigm in precision oncology. Semin Cell Dev Biol. (2019) pii: S1084-9521(18)30166-6. doi:10.1016/j.semcdb.2019.05.016.

[21] S. Aminzadeh-Gohari, D.D. WeberD, S. Vidali, L. Catalano, B. Kofler R.G. Feichtinger. From old to new - Repurposing drugs to target mitochondrial energy metabolism in cancer. Semin Cell Dev Biol. (2019) pii: S1084-9521(18)30188-5.

doi:10.1016/j.semcdb.2019.05.025. 